\title{
Evaluation of Environmental Requirements for Sustainable Ready-Mix Concrete Production in Abu Dhabi Emirate
}

\author{
Ghanim Kashwani' ${ }^{1}$, Abeer Sajwani' ${ }^{2}$, Muhammad Al Ashram², Rahma Al Yaaqoubi ${ }^{3}$ \\ ${ }^{1}$ Department of Civil Engineering, American University of Sharjah, Sharjah, UAE \\ ${ }^{2}$ RTI-International, Abu Dhabi, UAE \\ ${ }^{3}$ Abu Dhabi Company for Onshore Oil Operations, Abu Dhabi, UAE \\ Email: ghakas90@gmail.com, asajwani@ead.rti.org
}

Received 12 January 2014; revised 15 February 2014; accepted 11 March 2014

Copyright (C) 2014 by authors and Scientific Research Publishing Inc.

This work is licensed under the Creative Commons Attribution International License (CC BY). http://creativecommons.org/licenses/by/4.0/

(c) (i) Open Access

\section{Abstract}

Sustainability is the main concern for current and future construction projects in the world. In any project, attaining sustainability requires the integrity of four main elements. These elements cover economical, societal, cultural, and environmental aspects to its successful implementation and integration in an anthropogenic framework. Concrete is one of the main building materials used in current construction activities around the world; therefore, many issues pertaining to concrete long term sustainability arise. This makes issues related to environmental evaluation and assessment for the ready-mix concrete production process of vital importance. The release of particulate matter (PM) including PM10 and PM2.5 could be considered the main air pollutant that is emitted from this industrial sector; where PM can be released from different stages of ready-mix concrete production process. Another concern arising from the ready-mix concrete operations which may affect the soil and ground water quality is the spills or leaks that may occur from the storage containers used for diesel, motor oil, and chemical admixture. In 2010, Environmental Agency-Abu Dhabi (EAD) collected data pertaining to the operations of 73 ready-mix concrete facilities in the Emirate of Abu Dhabi. The data showed that the average production rate of concrete is around $250,000 \mathrm{~m}^{3}$ /year, which indicates that the concrete industry is a very active industry in the Emirate. Hence, EAD has established a set of plans and requirements to minimize the environmental impacts of concrete production on the environment and public health. The environmental impact of the concrete manufacturing can be reduced by conserving use of raw material ingredients, conserving energy consumption, and following Best Management Practices (BMPs) related to readymix concrete production. In this paper, a variety of BMPs are presented for ready-mix concrete manufacturing facilities in Abu Dhabi. These BMPs cover the following areas: raw materials handling and transport, process operation, housekeeping, waste management, emergency response, 
and training record. EAD can then regulate the ready-mix concrete manufacturing sector in Abu Dhabi using these BMPs and the requirements of the issued permitting conditions in order to have a sustainable industrial sector.

\section{Keywords}

Sustainability; Environmental Impacts; Air Quality; Best Management Practices

\section{Sustainability of Construction Materials}

The construction industry requires and consumes a lot of energy during the production and installation stages. To explain, $20 \%-25 \%$ of entire world energy is spent on construction materials such as cement, steel, concrete, etc. [1]-[3]. This energy can be consumed during extracting, processing, transporting, and assembling raw materials. This amount of consumed energy has short-term and long-term environmental effects that will be discussed later in the paper. Hence, conservation of energy and resources is needed to implement sustainability practices in the construction materials. Using new methods and technologies can be considered a very effective way to reduce the consumed energy of the construction material industry, which makes the construction materials more sustainable.

Becchio in his paper [1] mentions that there are many attempts to improve the sustainability of the concrete by using alternative fuels. For example, fuels such as biomass, tires, used oils, and used solvents, have a significant chance to enhance sustainability and reduce emissions and consumption of fossil fuels. To illustrate, using these alternative fuels can decrease the water/cement ratio in the concrete mix by having a good workability.

\section{Sustainability for the Concrete Industry}

To have a sustainable concrete industry, two aspects should be studied [4] [5]:

1) The durability of concrete

2) The environmental impacts of the concrete industry

For the concrete durability, it is essential to provide the full protection for the concrete structures from the different harsh environment factors that cause several degradation issues such as [1] [3]:

1) Creeping

2) Shrinkage

3) Random Cracks

Extreme conditions such as elevated temperatures and freeze/thaw cycles have a major significant impact in the concrete service life. In this region, there are two main factors that can affect the concrete structures; elevated temperature and elevated humidity.

Yehia explains in his paper [6], the mechanical behavior of various concrete (different aggregates integrates), the compressive strength and the elastic modules that can get affected during the fire. To explain, normal strength concrete (NSC) and high strength concrete (HSC) will have a major reduction in the compressive strength during a fire event before reaching to the critical temperature that cause spalling of the concrete. This is due to the buildup water pressure in the cement paste that decreases the loading bearing capacity and the concrete section. However, it should be noted that (HSC) has higher chance to spall than (NSC) because of the low permeability in its matrix. The author in his paper mentions that by using the lightweight aggregate, the concrete can sustain its compressive strength up to $1200^{\circ} \mathrm{F}$ and $60 \%$ of it elastic modulus at $800^{\circ} \mathrm{F}$.

Also, the author presents various suggestions to acquire better and more sustainable concrete. For example, using hybrid or composite concrete such as fiber reinforced concrete (FRC) can enhance the fire resistance feature comparing with the regular virgin concrete. To illustrate, different fibers such as polymers and steels are used in the cement paste to increase flexural and tensile strength. However, polypropylene fiber or mix with steel fiber could be used to reduce the effects of the steel fiber at elevated temperature [6].

The moisture damages on the concrete usually occur in the microstructure level. Microstructure damages in the concrete can be divided into two categories [7]:

1) Adhesive damage 
2) Cohesive damage (bonding damage)

Hossain says in his paper [7] that moist aggregate can have more effective adhesive and cohesive damages than the dry aggregate. The explanation behind that is due to the contact stress. The author conducted experimental test through finite element method (FRM) modeling to determine the behavior of matrix-aggregate interface (wet and dry) against contact stress and contact opening. It appears that, when the load is applied in the wet matrix, larger deformation occurs comparing with the dry matrix and that leads to reducing contact stresses and increasing contact opening in the wet matrix. The author concludes that the in wet condition, the adhesive and cohesive damages are more significant than in the dry condition.

Reducing the concrete permeability is the key solution to prevent the effects of moisture [7]. To explain, when the concrete has less permeability, the moisture flows with a very low rate and then causes less damage. There are three main factors that affect and control permeability in the concrete [8]:

1) The water cement ratio $(w / c)$

2) The cementitious materials

3) The size of the aggregate

Standard test like Rapid Chloride Test can help to evaluate the permeability level in the cement paste with respect to the water cement ratio (w/c). The following table shows the limits of acceptability for concrete permeability based on Rapid Chloride Test [9].

From Table 1, it can be inferred that when advance cementitous materials are used in the cement paste, less permeability occurs. This shows the important role of the cementitous materials in reducing moisture effects.

In general, cementitous materials decrease water demand, and reduce bleed channels that help to increase the concrete density and minimize the internal voids.

The next three points describe in details how the cementitous materials can improve the resistance of the concrete against moistures [8] [9]:

1) The cementitous material combines with calcium hydroxide which makes the concrete resistant to leaching

2) The cementitous material has lubricating effects that reduces the water in the cement paste and then reduce the internal voids and bleeding

3) Using cementitous material reduce the amount of calcium hydroxide which makes the concrete resistant to acids and salts effects.

For the aggregate size, it is advised to use fine or micro aggregate to fill the internal voids and then reduce the fluidity of the moistures inside the concrete [1].

\section{Environmental Impacts of Ready-Mixed Concrete Industry [10]}

In 2010, Environment Agency-Abu Dhabi (EAD) produced a concrete products manufacturing sector assessment report. The objective of this report was to characterize the environmental impacts and relative risks from the concrete products facilities operating in Abu Dhabi Emirate. Specifically, this report (1) provides a profile of the manufacturing processes and environmental impacts of the individual facilities within that sector using voluntarily reported data and (2) compares the mitigation strategies used by the sector to international best practices. EAD collected information from 73 ready-mix concrete facilities for the year 2009 and found that annual production of ready-mix concrete across the sector average 250,000 cubic meters per year. Sector-wide total dust emissions, or PM10, were estimated at 6163 metric tons per year.

The primary environmental and health concern in the ready-mix concrete (RMC) industry is dust. Dust reduces visibility, which may create driving hazards, and also leads to aesthetic damage, making buildings, statues,

Table 1. Chloride permeability based on charge passed.

\begin{tabular}{ccc}
\hline Charge Passed (coulombs) & Chloride Permeability & Typical of \\
\hline$>4000$ & High & High water/cement ratio (>0.6), PCC \\
$2000-4000$ & Moderate & Moderate water/cement ratio (0.4 to 0.5), PCC \\
$1000-2000$ & Low & Low water/cement ratio $(<0.4)$, PCC \\
$100-1000$ & Very Low & Latex-modified concrete, silica-fume concrete \\
$<100$ & Negligible & Polymer impregnated concrete, Polymer concrete \\
\hline
\end{tabular}


and cars look dirty. Dust particles can be carried over long distances by wind and then settle on ground or water. The effects of this settling can make lakes and streams acidic; change the nutrient balance in coastal waters and large river basins; deplete the nutrients in soil; damage sensitive forests and farm crops; and affect the diversity of ecosystems.

Several issues have been found across the ready-mix concrete sector during EAD inspections, including

- Inadequate filter maintenance on silos,

- Poor aggregate transfer practices that generate dust as the aggregate and other raw materials are transferred from area to another,

- General housekeeping issues such as not sweeping up dust in material storage areas.

\section{Best Management Practice for Ready-Mix Concrete Production Process [10]}

Rapid economic development and population growth is not yet complemented by fully effective environmental regulations and conditions for ready-mix concrete production industry. The challenges presented in this vital sector for development include the following:

1) Rapid population growth, fast industrialization and real estate development, have impacted the environment of the region in a harmful way.

2) Lack of legislation and awareness towards maintaining a sustainable ready-mix concrete production process within the industrial sectors.

3) The need for infrastructure has never been more crucial to control and support the ever increasing amount of ready-mix concrete being produced.

Therefore, EAD initiated Eltezam Campaign for ready-mix concrete manufacturing sector. The story behind the Eltezam campaign for the RMC manufacturing sector lies in an area called M-35 in Mussaffah Industrial Zone, Abu Dhabi. There are about 30 ready-mix concrete manufacturing facilities located in a relatively small geographic area of more than 70 hectare $\left(0.704 \mathrm{~km}^{2}\right)$. This huge concentration of RMC manufacturing facilities made daily operations at M-35 industrial plot unsafe due to the large amount of dust and PM emissions from the industrial operations. Some facilities are doing a good job in keeping basic housekeeping practices and following BMPs and even going beyond the scope of their facilities to clean up streets and remove track out; however, unfortunately this is not the case with the rest of the facilities. Thus, as the environmental regulator, Environment Agency —Abu Dhabi initiated the Eltezam Campaign for the ready-mix concrete manufacturing sector on October 8, 2013. The campaign's main goals are to raise awareness, reduce dust emissions, improve public health and safety and improve environmental compliance. About 160 ready-mix concrete manufacturing facilities operating around Abu Dhabi have been invited to a workshop that explained the main environmental impacts and best management practices of the industry. One month post the workshop, EAD environmental inspectors began visiting RMC facilities to check on the environmental compliance level. Inspections are still ongoing and effectiveness of the campaign will be measured through analyzing compliance level before and after the campaign [10].

The BMPs for the ready-mix concrete production process cover the following areas; raw materials handling and transport, process operation, housekeeping, waste management, emergency response, and training and record keeping. Through these specifically tailored BMPs for the ready-mix concrete manufacturing sector and the specific permit conditions issued for each operating ready-mix facility, EAD can regulate the ready-mix concrete production in order to achieve a sustainable industrial sector to work as an example for other operating industrial sectors.

Without proper controls and mitigation measures, process operations at ready-mix concrete facilities can adversely impact the environment and public health. But, BMPs can reduce environmental impacts from plant operations.

RMC plants generate large amounts of PM emissions because the primary raw materials used in the process are very dry and dusty.

- Portland cement is a very fine powder that easily becomes airborne.

- The aggregate used in making ready mix concrete also is dry and dusty.

- Most of the process activities that take place at ready-mix plants involve transporting or mixing these materials.

Other air pollutants are also emitted from fuel combustion in vehicles. Leaking containers and storage tanks, and spills can also lead to contamination of soil and groundwater. As stated before, the primary environmental 
issues and concerns at ready mix plants include: raw material loading, transfer, and handling of dry bulk materials, mixing operations, track-out of PM from vehicles, fuel combustion, vehicle washing, and equipment maintenance. The latter is of utmost importance; say for example, defective filters can cause large emission of cement dust from a storage silo.

Trackout is a serious problem for ready mix plants. Many ready mix plants wash their trucks and vehicles on site. Then, the wash water forms pools and mud. Concrete mixer trucks, aggregate delivery trucks, and other vehicles pick up the mud on their wheels. The mud is carried or "tracked" out of the plant and deposited onto nearby roads. The mud dries out and then becomes airborne when other vehicles drive over it.

Nearly all ready mix plants have diesel storage tanks that are used for refueling concrete mixer trucks and other vehicles. Diesel is sometimes spilled or leaked onto the ground when filling the storage tank or when refueling the trucks. Diesel, motor oil and other hazardous materials can also be spilled when vehicle maintenance is performed onsite. Moreover, chemical admixtures that are used in the concrete also can be spilled or can leak from storage containers.

For each hazard or potential release to the environment, there are one or more BMPs. Best Management Practices (BMPs) can be physical structures, activities, practices, or procedures that prevent, reduce, or mitigate an undesired event, impact, or effect. It is important to identify which BMPs apply to a situation. It is also important to understand which BMPs are the most appropriate for a hazard or environmental release. BMPs are organized by the following topic areas:

1) Raw Materials Handling, Storage, and Transfer

2) Process Operations

3) Housekeeping

4) Waste Management

5) Emergency Preparedness and Response

6) Training and Record Keeping

\subsection{BMPs for Raw Materials Handling, Storage, and Transfer}

Most of the raw materials used at ready mix plants are dry and dusty, so it is important to protect them from wind, vehicle traffic, and other factors that can cause them to be released to the environment as shown in Figure 1. All raw material storage piles should be protected from the wind using enclosures. Staff working at RMC should ensure that filters on cement silos are operating properly. Broken cement silo filters are one of the biggest problems observed at ready-mix facilities in the Emirate. Defective cement silo filters can lead to very large amounts of PM emissions. Usually, if you see a silo with cement dust on the top or down the silo that means the filter is not working.

Paving roadways and vehicle paths helps to minimize how much dust is released to the air as PM. Unpaveda

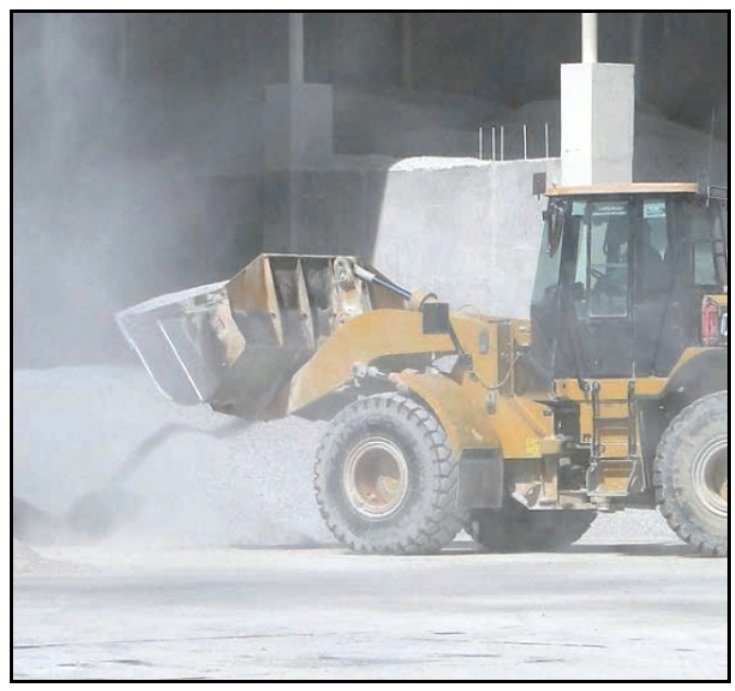

Figure 1. Bad handling of raw materials [10]. 
reas of the plant that are in vehicle paths should be sprayed with water to minimize emissions. Any raw material that is spilled should be cleaned up quickly before the material can become airborne. Moreover, the faster vehicles travel, the more dust becomes entrained and released, so it is important to keep vehicle speed down.

\subsection{BMPs for Process Operations}

The BMPs for process operations include the following:

- Use dust collection systems, such as baghouses and filters, to collect PM from handling and mixing operations

- Regularly inspect and maintain dust collection systems and bag houses

- Minimize number of material transfer points

- Minimize drop heights for material transfer to/from conveyors and hoppers

- Shroud or enclose transfer points

- Refuel vehicles on impervious surfaces

- Construct a bund wall around diesel storage tanks that contains $110 \%$ of the tank volume

- Monitor noise generated from operations

\subsection{BMPs for Housekeeping}

Housekeeping is an inexpensive way of preventing releases to the environment by removing dirt and other materials before they can cause problems. Housekeeping also eliminates hazards and makes the plant safer for employees and visitors. Housekeeping at RMC includes:

- Maintain clean premises and ground surfaces by sweeping, shoveling, wiping, and using dust collection vacuums

- Regularly schedule site cleanings to remove waste materials

- Provide vegetative barriers to reduce dust spreading

\subsection{BMPs for Waste Management}

There are 2 byproducts generated by the concrete industry: concrete washout waste material and washout wastewater. Concrete washout wastewater is a big problem given our proximity to the coastal environment and our low groundwater table. Concrete and cementitious washout wastewater is caustic, which means that it has a high $\mathrm{pH}$ (near 12). If allowed to run-off to surface waters, these washout wastes can change the $\mathrm{pH}$ of the water, which can, in turn, harm wildlife. It is best to deal with waste materials before they accumulate and cause serious problems. The steps that help to manage waste on the plant site include the following

- Perform truck washout activities in a concrete pit so that the wash water is not discharged onto the ground

- Collect, treat, and recycle washout wastewater, including concrete and cement (for example: mortar, grout, slurry) residues and contaminants, from truck and equipment washout activities

- Designate skips for solid waste materials

- Segregate hazardous and non-hazardous waste types

- Dispose of hazardous waste through an environmental service provider approved by the regulator.

- Collect and reuse waste concrete from the truck washout pit

- It is important not only to collect and remove waste, but to make sure the waste is properly disposed.

\subsection{BMPs for Emergency Response}

Spills, fires, and other emergencies can happen at any plant. The best way to minimize their effects is to be prepared. Every employee should be aware of what emergencies can happen and what they should do in response. Emergency response plans are critical for containing emergencies and ensuring the safety of employees, the public, and the environment. The BMPs for emergency response include:

- Provide spill prevention and response procedures and provide kits to clean up spills and leaks

- Label chemicals, and provide MSDS for each chemical

- Keep firefighting equipment properly maintained

- Provide appropriate PPE for employees

- Have an emergency response plan, train employees on emergencies, and conduct drills 


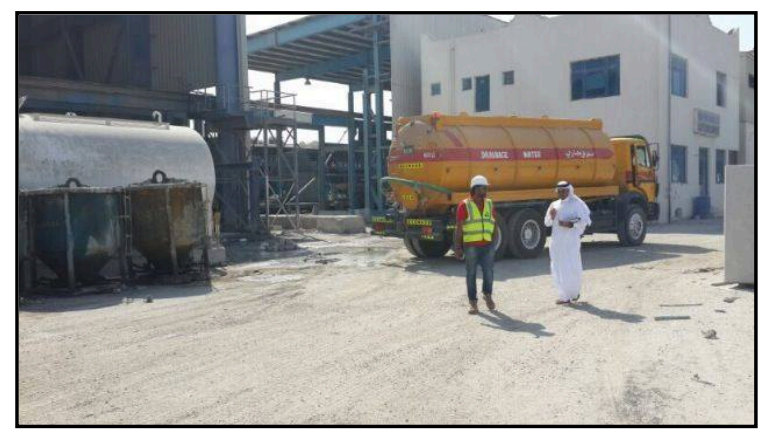

Figure 2. EAD regular inspection [10].

\subsection{BMPs for Training and Record Keeping}

In the event of an emergency, most employees want to do the right thing. But it is difficult if they do not know what they are supposed to do. That is why training is so important. Periodic refresher training courses are also important for ensuring that employees make good decisions when an emergency happens. Also, records are important for monitoring and controlling what happens on the plant site. The BMPs for training and record keeping include:

- Train employees in: good housekeeping, spill prevention and control, proper fueling procedures, safety, PPE, and material management

- Conduct induction and refresher training sessions regularly

- Provide an integrated EHSMS for the site, plant operations and delivery process

Maintain a complete environmental file with records such as Industrial Development Bureau (IDB)/EAD permit, civil defense certificate, training records, chemicals inventories and their MSDS, and inspections, audits and non-compliance reports (Figure 2).

\section{Conclusion}

Conserving use of raw material ingredients and energy can reduce the environmental effects of concrete manufacturing and lead to having a sustainable concrete industry. This can be done by following the new technologies and approaches in the ready-mix concrete filed such as advance concrete. Applying different (BMPs) related to ready-mix concrete production can minimize and contain the environmental impacts during the concrete manufacturing. Abu Dhabi Emirate can have more sustainable concrete industry by implementing these (BMPs).

\section{References}

[1] Becchio, C., Corgnati, S., Kindinis, A. and Pagliolico, S. (2009) Improving Environmental Sustainability of Concrete Products: Investigation on MWC Thermal and Mechanical Properties. Energy and Buildings, 41, 1127-1134. http://dx.doi.org/10.1016/j.enbuild.2009.05.013

[2] ACI Board Advisory Committee on Sustainable Development (2004) White Paper. ACI, Detroit.

[3] Mehta, K. (2001) Reducing the Environmental Impact of Concrete. Concrete International, 23, 61-65.

[4] Martin, N., Worrell, E. and Price, L. (1999) Energy Efficiency and Carbon Dioxide Emissions Reduction Opportunities in the US Cement Industry. Lawrence Berkeley National Laboratory, University of California.

[5] Martin, N., Worrell, E. and Price, L. (1999) Energy Efficiency and Carbon Dioxide Emissions Reduction Opportunities in the US Iron and Steel Sector. Lawrence Berkeley National Laboratory, University of California.

[6] Yehia, S. and Kashwani, G. (2013) Performance of Structures Exposed to Extreme High Temperature-An Overview. Open Journal of Civil Engineering, 3, 154-161. http://dx.doi.org/10.4236/ojce.2013.33018

[7] Hossain and Tarefder (2013) Effects of Moisture in Asphalt Concrete. Basic Research Journal of Engineering Innovation, 1, 16-25.

[8] US Department of the Interior, Bureau of Reclamation (1970) Fly Ash Increases Resistance to Sulfate Attack. Research Report No. 23, 5.

[9] Suprenant, BA. (1991) Testing for Chloride Permeability of Concrete. Concrete Construction.

[10] EAD (Environment Agency-Abu Dhabi). https://www.ead.ae/en/elibrary/ 